coloproctology $2018 \cdot 40: 100-101$ https://doi.org/10.1007/s00053-017-0223-7

Online publiziert: 19. Januar 2018

(c) Springer Medizin Verlag GmbH, ein Teil von Springer Nature 2018

CrossMark

\title{
H. Feußner
}

Klinikum rechts der Isar, Klinik und Poliklinik für Chirurgie, Technische Universität München, München, Deutschland

\section{Computerunterstützte Chirurgie}

\section{Mehr als nur Robotik!}

Dass im Rahmen der zunehmenden Digitalisierung aller Lebensbereiche auch die Chirurgie einem tief greifenden Wandel ausgesetzt sein wird, ist nicht mehr nur einer kleinen Gruppe von technikaffinen Avantgardisten bewusst, sondern scheint jetzt bei der Mehrheit der Chirurgen anzukommen. Dieser Wandel wird immer häufig auf den Kongressen und in den Publikationsorganen thematisiert, wobei sehr häufiger nur auf das Thema „Robotik" fokussiert wird. Abgesehen davon, dass das, was in der Chirurgie als „Robotereinsatz" apostrophiert wird, eigentlich ein Etikettenschwindel ist (tatsächlich handelt es sich nur um sog. Master-Slave-Systeme), ist der Einsatz von da Vinci oder ähnlichen Systemen zwar das spektakulärste, aber eigentlich das unbedeutendste Anwendungsbeispiel für die computerunterstützte Chirurgie. Viel entscheidender sind die Impulse, die aus einer ganz anderen Richtung kommen.

\section{》) Computerunterstützung wird auch in der Durchführung der chirurgischen Behandlung immer wichtiger}

Tatsächlich umfasst die „computerunterstützte Chirurgie“ eine sehr breite Palette von Anwendungsmöglichkeiten, deren gemeinsamer Nenner die intelligente Informationsgewinnung und -nutzung ist. Computerunterstützte Chirurgie ist aber nicht nur Software, sondern schließt natürlich auch die Nutzung von Maschinen und Gerätesystemen ein, deren Funktion von modernen informationsverarbeitenden Systemen abhängt.

Computerunterstützung spielt nicht nur in der chirurgischen Diagnostik und Entscheidungsfindung, sondern auch in der Organisation und Durchführung der chirurgischen Behandlung eine immer wichtigere Rolle. In der Diagnostik und Entscheidungsfindung spannt sich der Bogen von der verbesserten Visualisierung (z. B. durch die 3-DVideodarstellung) über die Sichtbarmachung von Pathologien (intraoperative Gewebsdifferenzierung) bis hin zur verbesserten Wahrnehmung des chirurgischen Situs durch die Kombination von präoperativ gewonnenen Schnittbildern mit dem intraoperativen Situs. Die beeindruckenden Fortschritte resultieren aus bahnbrechenden Entwicklungen in den Natur- und Ingenieurwissenschaften sowie der Informatik, beginnend mit immer leistungsfähigeren Sensoren, präziseren Navigationsverfahren, Bildverarbeitungsverfahren bis hin zu anspruchsvollen neuen Ansätzen der Datenverarbeitung. Am Ende führen sie - so ist jedenfalls zu hoffen - auch $\mathrm{zu}$ einer verbesserten, effizienteren und sichereren Therapie.

Die vier Beiträge in dieser Ausgabe sollen die Thematik dem Leser von coloproctology näherbringen, wobei wir uns bewusst sind, dass die Beschäftigung mit dieser teilweise abstrakten und vielleicht derzeit noch sehr naturwissenschaftlichtechnisch geprägten Wissensdomäne Mühe abverlangt. Wir müssen als Chirurgen aber die Energie aufbringen, uns mit den Herausforderungen der computerunterstützten Chirurgie auseinanderzusetzen - bevor es andere für uns tun.

Wilhelm et al. bringen in ihrem Beitrag zunächst einmal eine terminologische Klärung und präsentieren eine verbindliche Definition dessen, was man als „computerbasierte Chirurgie“ bezeich- 
net: „Computerbasierte Chirurgie' ist die konsequente Nutzung allen vorhandenen Wissens und die Entwicklung von neuen Methoden des Erkenntnisgewinns, um die diagnostische und therapeutische Entscheidung einschließlich der Behandlungsdurchführung selbst sicherer, schneller und im Gesamtergebnis des Behandlungsprozesses besser zu machen." In ihrer Hierarchie der Begrifflichkeiten ordnen die Autoren die heute meist ziemlich unscharf verwendeten Schlagwörter ihrer korrekten Bedeutung nach ein und schaffen so die Voraussetzung für eine wissenschaftliche Diskussion der computerassistierten Chirurgie. Darüber hinaus skizzieren sie konkret die fast atemberaubenden Chancen, die sich für die Chirurgie eröffnen. Die Gewinnung akademischen Nachwuchses ist dabei ein nicht zu unterschätzender Aspekt. Allerdings ist dies für uns Chirurgen auch mit ganz konkreten Herausforderungen verbunden, denen wir uns unbedingt stellen müssen. Es geht längst nicht mehr nur um die klinische Evaluation von Prototypen, auf die wir uns in der Vergangenheit meist beschränkt haben. Künftig müssen wir uns bereits in die Ideenfindung und in die Entwicklungsbegleitung einbringen, damit die Translation neuer Konzepte „from bench to bedside“ schneller und zielgerichteter abläuft.

Im zweiten Beitrag untersuchen K. Krajinovic u. M. Kim konkret, welchen Mehrwert „Robotik“-Anwendungen in der Chirurgie derzeit tatsächlich mit sich bringen. In einer sehr sorgfältigen Analyse der heute zur Verfügung stehenden Literatur unter besonderer Berücksichtigung der onkologischen kolorektalen Chirurgie zeigen sie, dass die da-Vinci-unterstützte Chirurgie dem laparoskopischen Vorgehen zumindest nicht unterlegen ist. Allerdings ist - trotz aller technischen Vorteile des Telemanipulationssystems - auch kein eindeutiger Vorteil hinsichtlich der postoperativen Funktionseinschränkungen oder des onkologischen Ergebnisses belegt. Demgegenüber sind die Operationszeiten länger und die Kosten höher, wobei die Hoffnung geäußert wird, dass sich diese nach Verlust der Monopolstellung des bisher einzigen Herstellers reduzieren werden. Demgegenüber besteht zweifellos ein ergonomischer Vorteil für den Operateur - aber eben nicht für alle Mitglieder des Operationsteams. Der Einsatz von Telemanipulatoren in der Chirurgie ist aber kein Wert an sich, sondern eigentlich nur dann vertretbar, wenn sich daraus in erster Linie ein messbarer Vorteil für den Patienten ergibt. Angesichts der großen Zahl von Untersuchungen und Metaanalysen und auch qualitativ hochwertigen Studien, wie der auch hier zitierten ROLARR-Studie, ist es eigentlich müßig, noch gebetsmühlenartige weitere Studien zu fordern. Konsequent weisen die Autorinnen deshalb auf das große Zukunftspotenzial hin, das die künftigen Varianten und Neuentwicklungen der Telemanipulationssysteme haben.

\section{》) Lernfähige Assistenzsysteme sind keine Angelegenheit der fernen Zukunft}

Bevor dies im Einzelnen von Koller et al. dargestellt wird, skizzieren Speidel et al. in ihrem Beitrag „Kognitive Chirurgie/ Chirurgie 4.0“ den übergeordneten Rahmen, in dem die oben erwähnten Telemanipulationssysteme der nächsten Generation ihr volles Potenzial entfalten können. Die Chirurgie wird künftig ergänzt werden durch technisch-kognitive Systeme, die nicht nur vorgegebene Aufgaben durchführen, wie etwa unsere heutigen „OP-Roboter“, sondern eine gegebene Situation verstehen, angepasst handeln und daraus auch - wie ein menschlicher Assistent - lernen. Dass dies nicht Angelegenheit der fernen Zukunft ist, sondern in mittelbarer Zeit Realität werden kann, zeigt dieser Beitrag überzeugend.

Vor diesem Hintergrund können die disruptiven Entwicklungen bei mechatronischen Assistenzsystemen und Telemanipulatoren realisiert werden, die im letzten Beitrag von Koller et al. konkret und anschaulich dargestellt werden. Die faszinierenden Entwicklungen implizieren aber auch erhebliche Herausforderungen an die Chirurgen, auf die ja bereits Wilhelm et al. hingewiesen haben. Wir müssen unsere Eingriffe künftig besser standardisieren, modellieren und evi- denzbasiert gestalten. Unser spezifisch chirurgisches Wissen muss so aufbereitet werden, dass es von einem technischkognitiven System „verstanden“ werden kann - um nur einige wenige dringende Aufgaben zu nennen. Sich darum konsequent zu kümmern, wäre bei unseren begrenzten wissenschaftlichen Ressourcen jedenfalls sinnvoller, als in zahlreichen weiteren Studien zu untersuchen, ob sich die „Roboterchirurgie“ nicht vielleicht doch in kleinen Subgruppen rechtfertigen lässt.

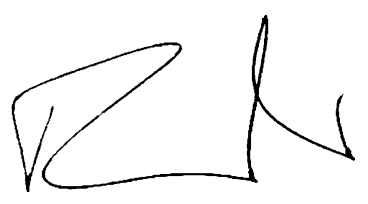

\section{H. Feußner}

\section{Korrespondenzadresse

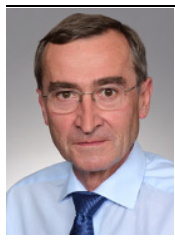 \\ Prof. Dr. H. Feußner \\ Klinikum rechts der Isar, Klinik und Poliklinik für Chirurgie, Technische Universität München \\ Ismaninger Str. 22, 81675 München, Deutschland hubertus.feussner@tum.de}

Interessenkonflikt. H. Feußner gibt an, dass kein Interessenkonflikt besteht. 